\title{
Aplicação da Formulação de Mínimos Quadrados a Problemas de Difusão
}

R.C.P. LEAL-TOLEDO ${ }^{1}$, Departamento de Ciência da Computação, Instituto de Computação, Universidade Federal Fluminense, Rua Passo da Pátria 156, 24210-240 Niterói, RJ, Brasil

E.M. TOLEDO ${ }^{2}$, Laboratório Nacional de Computação Científica, Av. Getúlio Vargas 333, 25651-070 Petrópolis, RJ, Brasil

M.S. VASCONCELOS, Pós-Graduação em Ciência da Computação, Universidade Federal Fluminense, RJ, Brasil.

\begin{abstract}
Resumo. Apresentam-se, neste trabalho, formulações mistas de mínimos quadrados para o problema de difusão transiente, como alternativa à formulação clássica de elementos finitos, via método de Galerkin. Aplicadas ao problema de Poisson estacionário, descrito como um problema misto, obtém-se convergência na norma de $H^{1}$ para a grandeza escalar e em $H_{\text {div }}$ para seu fluxo, sem necessidade de compatibilidade entre os espaços que aproximam essas variáveis. Quando a condição de irrotacionalidade do fluxo é acrescentada, consegue-se convergência na norma de $H^{1}$ para ambas variáveis envolvidas no problema.

Tendo em vista esses resultados, propõem-se formulações semi-discretas de mínimos quadrados, considerando-se também o problema como misto. Diferentes ponderações no tempo são utilizadas para definir funcionais de mínimos quadrados em diferentes instantes de tempo. A influência do acréscimo da equação relativa ao rotacional nulo nesses funcionais é testada.

Exemplos numéricos confirmam as taxas de erro encontradas para o problema estacionário e mostram a convergência das formulações propostas para o problema transiente.
\end{abstract}

\section{Introdução}

O método dos elementos finitos é, sem dúvida, a ferramenta mais utilizada na resolução de problemas de valor de contorno. Neste contexto, formulações de Galerkin em um campo, mostram-se eficientes em aplicações de diversas áreas. Entretanto, em vários problemas de interesse, constatam-se comportamentos indesejáveis, tais como, oscilações espúrias e trancamento de soluções. O que tem sido proposto para

\footnotetext{
${ }^{1}$ leal@dcc.ic.uff.br

2 emtc@lncc.br, Universidade Federal de Juiz de Fora.
} 
estes casos é a utilização de formulações mistas baseadas no método de Galerkin e mais recentemente, as formulações de Petrov-Galerkin, obtidas pela adição de resíduos de mínimos quadrados de suas equações à forma variacional do problema.

Paralelamente, tem surgido na literatura propostas de utilização da formulação de mínimos quadrados. Essa formulação que em sua forma clássica tem a desvantagem de requerer maior grau de regularidade dos espaços de aproximação, se comparada a outras formulações, foi estudada por Bramble e Schatz [1] na solução numérica de problemas de valor de contorno elípticos. A transformação da equação diferencial em um sistema de equações diferenciais equivalentes de primeira ordem, é utilizada para reduzir essa necessidade adicional de regularidade desses espaços de aproximação. Diversos autores adotam esse tipo de abordagem para tratar problemas tanto estacionários [3],[4],[7],[9] quanto transientes [5],[8].

Neste trabalho propõem-se formulações deste tipo aplicadas a sistemas de primeira ordem derivados do problema de Poisson, tanto estacionário quanto transiente, formulados nas variáveis escalar e vetorial.

Para o problema estacionário apresenta-se a análise de existência e unicidade de solução e as taxas de convergência encontradas em [10], [9], inclusive acrescentandose às equações do problema a condição de irrotacionalidade do fluxo. Resultados teóricos similares foram apresentados em [3] e [11] e posteriormente generalizados para problemas de advecção-difusão-reação em [2] a partir de resultados preliminares obtidos por Girault-Raviart[6]. Resultados numéricos comprovam as taxas de convergência obtidas nessas análises.

Para o problema transiente, propõem-se formulações semi-discretas a partir da definição de funcionais de mínimos quadrados em diferentes instantes de tempo. Dessa forma, apresenta-se uma formulação totalmente implícita onde todas as variáveis estão descritas no tempo $t+\Delta t$ e uma formulação ponderada, onde a equação evolutiva foi ponderada por um fator $\theta$ entre os tempos $t$ e $t+\Delta t$ e a equação constitutiva, que relaciona a grandeza escalar a seu fluxo, no tempo $t+\Delta t$. Também nesses casos, acrescenta-se a condição de irrotacionalidade do fluxo para melhorar as taxas de convergência para essa variável. Apresentam-se resultados numéricos que permitem verificar a convergência dos esquemas propostos, analisando-se a influência do acréscimo da equação do rotacional nulo.

\section{Definição do Problema}

Seja $\Omega \in R^{n s d}(n s d=1,2)$ um domínio aberto de contorno $\Gamma, \mathbf{x}=\left(x_{1}, x_{2}\right)$ um ponto em $\Omega$ e $\mathbf{n}=\left(n_{1}, n_{2}\right)$ o vetor normal ao contorno. Considera-se a seguinte equação diferencial de segunda ordem :

$$
\begin{array}{rll}
u_{t}-\nabla \cdot(\mathbf{k} \nabla u)=f(\mathbf{x}, t) & \text { em } \quad & \Omega, \\
u=g(\mathbf{x}, t) & \text { em } \quad \Gamma_{u}, \\
\mathbf{p} \cdot \mathbf{n}=h(\mathbf{x}, t) & \text { em } \quad \Gamma_{q}, \\
u=u_{0}(\mathbf{x}) & \text { em } & \Omega \text { para } t=0,
\end{array}
$$


com $\Gamma_{u} \cup \Gamma_{q}=\Gamma$ e $\Gamma_{u} \cap \Gamma_{q}=\emptyset$, onde $u$ é a variável escalar, $\mathbf{p}$ é seu fluxo, e $f(\mathbf{x}, t) \in L^{2}(\Omega)$ e $g(\mathbf{x}, t)$ funções dadas.

Como a formulação aqui apresentada é aplicada a um sistema de equações de $1^{a}$ ordem, reescreve-se o problema descrito pelas equações (2.1) como:

$$
\begin{array}{rll}
u_{t}+\operatorname{div} \mathbf{p}=f(\mathbf{x}, t) & \text { em } \quad \Omega, \\
\mathbf{p}=-\mathbf{k} \nabla u & \text { em } \quad \Omega, \\
u=g(\mathbf{x}, t) & \text { em } \quad \Gamma_{u}, \\
\mathbf{p} \cdot \mathbf{n}=h(\mathbf{x}, t) & \text { em } \quad \Gamma_{q}, \\
u=u_{0}(\mathbf{x}) & \text { em } \quad \Omega \text { para } t=0,
\end{array}
$$

onde $\nabla$ é o operador gradiente e $\operatorname{divp}=\nabla \cdot \mathbf{p}$ o divergente do fluxo e $\mathbf{k}$ é uma matriz dada por $\mathbf{k}=k \mathbf{I}$ sendo $\mathbf{I}$ a matriz identidade, e $k$ uma constante.

A seguir, definem-se alguns espaços de funções e normas associadas, necessárias ao desenvolvimento do presente trabalho. Seja $L^{2}(\Omega)$ o espaço das funções quadrado integráveis em $\Omega$ com produto interno definido da forma usual como $(u, v)=$ $\int_{\Omega}(u v) d \Omega$ e norma $\|u\|_{0}=\|u\|=(u, u)^{\frac{1}{2}}$ e $H^{1}(\Omega)$ o espaço de Hilbert $H^{1}(\Omega)=$ $\left\{u \in L^{2}(\Omega) \forall \alpha,|\alpha| \leq 1,\left(\partial^{\alpha} u\right) \in L^{2}(\Omega)\right\}$. Em particular, definem-se ainda os seguintes espaços:

$$
\begin{gathered}
V=\left\{v \in H^{1}: v=0 \quad \text { em } \quad \Gamma_{u}\right\} \\
U=\left\{\mathbf{q} \in L^{2}(\Omega)^{n}: \operatorname{div} \mathbf{q} \in L^{2}(\Omega)\right\} \equiv H(\operatorname{div}),
\end{gathered}
$$

as normas: $\|\mathbf{q}\|_{H(\text { div })}^{2}=\|\mathbf{q}\|^{2}+\|\operatorname{divq}\|^{2}$ e $\|\mathbf{q}\|_{H^{1}}^{2}=\|\mathbf{q}\|^{2}+\|\nabla \mathbf{q}\|^{2}$, e a norma no espaço produto: $\|(\mathbf{q}, v)\|_{H(\operatorname{div}) \times H^{1}}=\left(\|\mathbf{q}\|_{H(\operatorname{div})}^{2}+\|v\|_{H^{1}}^{2}\right)^{\frac{1}{2}}$.

\section{O Problema Estacionário}

Define-se o problema estacionário, com condições de contorno homogêneas de Dirichlet, como:

Problema P1: Para um dado campo $f$ escalar, achar os campos $\mathbf{p}$ e $u$ que satisfaçam a:

$$
\begin{array}{rll}
\operatorname{divp}=f & \text { em } & \Omega, \\
\mathbf{p}=-\mathbf{k} \nabla u & \text { em } & \Omega, \\
u=\bar{u}=0 & \text { em } & \Gamma_{u} \equiv \Gamma .
\end{array}
$$

O funcional de mínimos quadrados correspondente ao Problema P1 é dado por:

$$
J(\mathbf{q}, v)=\frac{1}{2} \int_{\Omega}\left[(\operatorname{div} \mathbf{q}-f)^{2}+(\mathbf{q}+\mathbf{k} \nabla v)^{2}\right] d \Omega .
$$

Associado à minimização de $J(\mathbf{q}, v)$, tem-se o seguinte problema variacional: 
Problema V1: Achar $\{\mathbf{p}, u\} \in U \times V$ tal que:

$$
B(\{\mathbf{p}, u\},\{\mathbf{q}, v\})=F(\mathbf{q}, v), \quad \forall \mathbf{q} \in U, \quad v \in V,
$$

com $B(\cdot, \cdot)$ e $F(\cdot)$ definidas como:

$$
\begin{aligned}
B(\{\mathbf{p}, u\},\{\mathbf{q}, v\})= & (\operatorname{div} \mathbf{p}, \operatorname{div} \mathbf{q})+(\mathbf{p}+\mathbf{k} \nabla u, \mathbf{q}+\mathbf{k} \nabla v), \\
& \mathbf{F}(\mathbf{q})=(f, \operatorname{div} \mathbf{q}) .
\end{aligned}
$$

Apesar da formulação estar definida em dois campos, existência e unicidade de solução para o problema contínuo são dadas pelo lema de Lax-Milgram uma vez que o operador $B(\cdot, \cdot)$ definido em (3.1) é contínuo e coercivo, isto é:

$$
\begin{gathered}
\|B(\{\mathbf{p}, u\},\{\mathbf{q}, v\})\| \leq M\|\mathbf{q}, v\|_{H(\operatorname{div}) \times H_{1}}\|\mathbf{p}, u\|_{H(\operatorname{div}) \times H_{1}}, \\
B(\{\mathbf{q}, v\},\{\mathbf{q}, v\}) \geq \alpha\|\mathbf{q}, v\|^{2}{ }_{H(\operatorname{div}) \times H_{1}},
\end{gathered}
$$

com $M$ e $\alpha>0$, sendo $\alpha=\min \left\{\alpha_{1}, \alpha_{2}\right\}$, onde $\alpha_{1}=\min \{\epsilon, 1-\epsilon \kappa\}$ e $\alpha_{2}=$ $\min \left\{\frac{\epsilon}{2}, \epsilon\left\{\frac{c}{2}-\frac{1}{\kappa}\right\}\right\} \operatorname{com} \epsilon \in(0,1)$ e $\kappa>0[9],[10]$.

Análise numérica

Para introduzir uma aproximação por elementos finitos para o Problema V1, considera-se $H_{h}^{k} \subset H$ (div) e $V_{h}^{l} \subset H^{1}$, onde $k$ e $l$ são os graus dos polinômios de interpolação para $\mathbf{p}$ e $u$, respectivamente, e $h$, um parâmetro da malha.

Problema $V 1_{h}$ : Calcular $\left(\mathbf{p}_{h}, u_{h}\right) \in H_{h}^{k} \times V_{h}^{l}$ tal que :

$$
B\left\{\left(\mathbf{p}_{h}, u_{h}\right),\left(\mathbf{q}_{h}, v_{h}\right)\right\}=F\left(\mathbf{q}_{h}, v_{h}\right), \quad \forall \mathbf{q}_{h} \in H_{h}^{k} \text { e } \forall v_{h} \in V_{h}^{l} .
$$

i. Existência, unicidade e consistência para o problema aproximado decorrem imediatamente da existência e unicidade da solução do Problema $V 1$ e de que $H_{h}^{k} \subset$ $H(\operatorname{div})$ e $V_{h}^{l} \subset H^{1}$.

ii. A ortogonalidade do erro no espaço $H_{h}^{k} \times V_{h}^{l}$ decorre imediatamente da definição de $B(\cdot, \cdot)$ e da consistência, garantindo que o erro é ortogonal ao subespaço de aproximações.

iii. Melhor aproximação e estimativa de erro - devido à simetria de $B(\cdot, \cdot)$ e considerando a ortogonalidade do erro, a elipticidade e continuidade de $B(\cdot, \cdot)$, pode-se concluir que:

$$
\left\|\left(\mathbf{p}-\mathbf{p}_{h}\right),\left(u-u_{h}\right)\right\|_{H(\operatorname{div}) \times H^{1}} \leq \sqrt{\frac{M}{\alpha}}\left\|\left(\mathbf{p}-\mathbf{q}_{h}\right),\left(u-v_{h}\right)\right\|_{H(\operatorname{div}) \times H^{1}} .
$$

Tendo-se em vista (3.2), as propriedades de interpolação nos espaços $H$ (div) e $H^{1}$ e, admitindo-se que a solução exata $\{\mathbf{p}, u\}$ seja suficientemente regular com $f \in L^{2}(\Omega)$, obtém-se, para $k=l$, as seguintes estimativas:

$$
\left\|\mathbf{p}-\mathbf{p}_{h}\right\|_{H(\mathrm{div})}+\left\|u-u_{h}\right\|_{H^{1}} \leq C h^{k},
$$

que são as mesmas ordens de aproximação para $\mathbf{p}$ e para $u$ que as conseguidas pelo método de Petrov-Galerkin [12]. Resultados semelhantes podem ser encontrados em [11] quando $\mathbf{k}$ é uma matriz diagonal com coeficientes variáveis. 


\subsection{Uma Formulação com Rotacional}

Apresenta-se, nesta seção, uma formulação que inclui a condição de irrotacionalidade do fluxo ( $\operatorname{rot} \mathbf{p}=0$ ), decorrente de $\mathbf{p}=-k \nabla u$. O funcional de mínimos quadrados, neste caso, é dado por:

$$
\bar{J}(\mathbf{q}, v)=J(\mathbf{q}, v)+\frac{1}{2} \int_{\Omega}(\operatorname{rot} \mathbf{q})^{2} d \Omega .
$$

Associado à minimização de $\bar{J}(\mathbf{q}, v)$, tem-se o seguinte problema variacional: Problema V2: Achar $(\mathbf{p}, u) \in W \times V$, tal que:

$$
\bar{B}\{(\mathbf{p}, u),(\mathbf{q}, v)\}=F(\mathbf{q}, v), \quad \forall \mathbf{q} \in W, \quad v \in V
$$

$\operatorname{com} \bar{B}(\cdot, \cdot)$ e $F(\cdot)$ definidas a seguir.

$$
\begin{gathered}
\bar{B}\{(\mathbf{p}, u),(\mathbf{q}, v)\}=B\{(\mathbf{p}, u),(\mathbf{q}, v)\}+(\operatorname{rot} \mathbf{p}, \operatorname{rot} \mathbf{q}), \\
\mathbf{F}(\mathbf{q})=(f, \operatorname{div} \mathbf{q}) .
\end{gathered}
$$

Existência e unicidade de solução

Pelo Lema de Lax, as provas de existência e unicidade reduzem-se às provas da continuidade e elipticidade de $B(\cdot, \cdot)$. Adotando-se um procedimento semelhante ao utilizado para o Problema V1 e utilizando-se a relação de equivalência [6],

$$
\|\operatorname{div} \mathbf{q}\|^{2}+\|\operatorname{rot} \mathbf{q}\|^{2}+\|\mathbf{q}\|^{2}=\|\mathbf{q}\|_{W}^{2} \geq C_{1}\|\mathbf{q}\|_{1}^{2}, \quad \forall \mathbf{q} \in W \equiv H^{1},
$$

pode-se garantir que: $\bar{B}\{(\mathbf{q}, v),(\mathbf{q}, v)\} \geq \alpha\|(\mathbf{q}, v)\|_{H^{1} \times H^{1}}^{2}$ ficando assegurada a elipticidade de $B(\cdot, \cdot)$ em $W \times V$, com $W \equiv V \equiv H^{1}$, sendo a norma do espaço produto definida como: $\|(\mathbf{q}, v)\|_{H^{1} \times H^{1}}^{2}=\|\mathbf{q}\|_{1}^{2}+\|v\|_{1}^{2}$. A continuidade da forma $B(\cdot, \cdot)$ pode ser demonstrada para $\bar{M} \geq 6$, chegando-se a:

$$
|\bar{B}\{(\mathbf{p}, u),(\mathbf{q}, v)\}| \leq M\|(\mathbf{p}, u)\|_{H^{1} \times H^{1}}\|(\mathbf{q}, v)\|_{H^{1} \times H^{1}} .
$$

Análise numérica

Problema $V 2_{h}$ : Calcular $\left(\mathbf{p}_{h}, u_{h}\right) \in W_{h}^{k} \times V_{h}^{l}$ tal que :

$$
\bar{B}\left\{\left(\mathbf{p}_{h}, u_{h}\right),\left(\mathbf{q}_{h}, v_{h}\right)\right\}=F\left(\mathbf{q}_{h}, v_{h}\right), \quad \forall \mathbf{q}_{h} \in W_{h}^{k} \subset H^{1} \text { e } \forall v_{h} \in V_{h}^{l} \subset H^{1} .
$$

Da mesma forma que no Problema $V 1_{h}$, pode-se garantir a ortogonalidade do erro e a existência e unicidade da solução do Problema $V 2_{h}$. O Lema de Céa, é expresso por: $\left\|\left(\mathbf{p}-\mathbf{p}_{h}\right),\left(u-u_{h}\right)\right\|_{H^{1} \times H^{1}} \leq C\left\|\left(\mathbf{p}-\mathbf{q}_{h}\right),\left(u-v_{h}\right)\right\|_{H^{1} \times H^{1}}$ que, combinado com os resultados da teoria de interpolação, fornece as seguintes estimativas:

$$
\left.\left\|\left(\mathbf{p}-\mathbf{p}_{h}\right)\right\|_{0}+h \| \nabla \mathbf{p}-\nabla \mathbf{p}_{h}\right)\left\|_{0}+\right\| u-u_{h}\left\|_{0}+h\right\| \nabla u-\nabla u_{h} \|_{0} \leq C h^{k+1}
$$

ou $\left\|\mathbf{p}-\mathbf{p}_{h}\right\|_{1} \leq C h^{k}$ e $\left\|u-u_{h}\right\|_{1} \leq C h^{k}$. Neste caso, para $l=k$, as estimativas na norma de $L^{2}$ são da ordem de $h^{k+1}[9]$, como será visto no exemplo a seguir. Análise semelhante é apresentada em [11] e [3] quando a matriz k é diagonal com coeficientes variáveis e generalizada para o caso de $\mathbf{k}$ não diagonal em [2]. 


\subsection{Resultados Numéricos}

Para confirmar as taxas de convergências apresentadas, considera-se um exemplo simples, com solução exata conhecida, que consiste em um problema definido em um domínio quadrado $\Omega=[0,1] \times[0,1]$, com condições de contorno, $u=0$ em $\Gamma$ e com solução exata $u=\operatorname{sen} \pi x \operatorname{sen} \pi y$.

Adotam-se malhas uniformes de elementos finitos, com 2x2, 4x4, 8x8 e 16x16 elementos isoparamétricos, bilineares com interpolação de igual ordem $(k=l=1)$. Na Figura 1, apresentam-se as taxas de convergência obtidas com e sem a inclusão do rotacional nulo nas equações do problema.

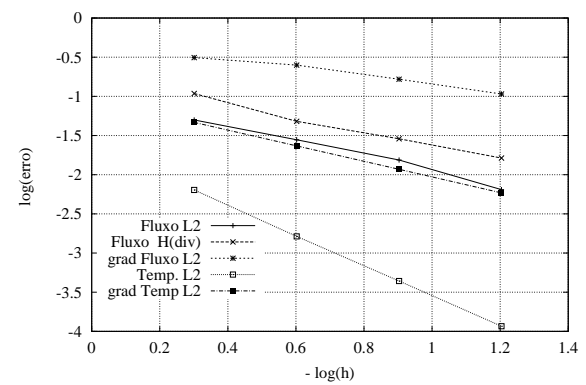

$(a)$

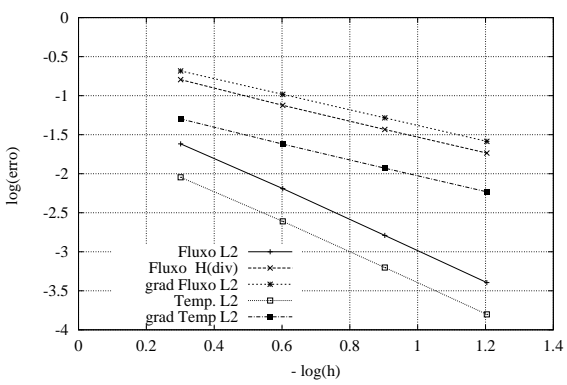

$(b)$

Figura 1: Convergência para o caso estacionário a) Sem rotacional b) Com rotacional

Além de confirmar os resultados encontrados na análise, pode-se observar que a inclusão da parcela devida ao rotacional recupera a convergência do fluxo em $L^{2}$ que se apresenta com erro na ordem de $h^{2}$.

\section{O Problema Transiente}

Para o tratamento do problema transiente, considera-se $I$ um intervalo de tempo $I=(0, T)$ com partição $0=t_{0}<t_{1}<\cdots<t_{N}=T, I_{n} \operatorname{como} I_{n}=\{(\mathbf{x}, t)$ : $\left.\mathbf{x} \in \Omega, t_{n}<t<t_{n+1}\right\}$ e $t_{n+\theta}$ como um instante de tempo com $t_{n}<t_{n+\theta} \leq$ $t_{n+1}$ para $n=0,1, \ldots, N-1$. Discretizando-se a equação evolutiva do sistema (2.2) pelo método das diferenças finitas no tempo $t_{n+\theta}$ e considerando-se a equação constitutiva no instante de tempo $n+1$ tem-se:

Problema P2: Para $n=1,2, \ldots, N-1$ e $\theta \in(0,1]$ e para um dado campo escalar $f$, encontrar os campos $\mathbf{p}$ e $u$ que satisfaçam a:

$$
\begin{aligned}
\frac{u^{n+1}-u^{n}}{\Delta t}+\operatorname{div} \mathbf{p}^{n+\theta} & =f^{n+\theta} \mathrm{em} \Omega, \\
\mathbf{p}^{n+1} & =-\mathbf{k} \nabla u^{n+1},
\end{aligned}
$$


com $u=g(\mathbf{x}, t)$ em $\Gamma_{u}=\Gamma$ e $u=u_{0}(\mathbf{x})$ em $\Omega$ para $t=0$. O funcional de mínimos quadrados para este sistema de equações é dado por:

$$
\begin{aligned}
J(\mathbf{q}, v)=\frac{1}{2}\left[\left(\frac{v^{n+1}-v^{n}}{\Delta t}+\operatorname{div} \mathbf{q}^{n+\theta}\right.\right. & \left.-f^{n+\theta}, \frac{v^{n+1}-v^{n}}{\Delta t}+\operatorname{div} \mathbf{q}^{n+\theta}-f^{n+\theta}\right) \\
& \left.+\left(\mathbf{k} \nabla v^{n+1}+\mathbf{q}^{n+1}, \mathbf{k} \nabla v^{n+1}+\mathbf{q}^{n+1}\right)\right] .
\end{aligned}
$$

Para permitir aproximações em diferentes instantes de tempo $t_{n+\theta}$, substituem-se em (4.2) $v$ e $\mathbf{q}$ por suas aproximações $v_{h}$ e $\mathbf{q}_{h}$ respectivamente, utilizando uma ponderação para aproximar $\mathbf{q}_{h}^{n+\theta}$ e $f^{n+\theta}$ na equação evolutiva. Definem-se, então, as seguintes ponderações: $\mathbf{q}_{h}^{n+\theta}=\theta \mathbf{q}_{h}^{n+1}+(1-\theta) \mathbf{q}_{h}^{n}$ e $f^{n+\theta}=\theta f^{n+1}+(1-\theta) f^{n}$. Dessa forma, o funcional de mínimos quadrados obtido é dado por:

$$
\begin{aligned}
J\left(\mathbf{q}_{h}, v_{h}\right)=\frac{1}{2}[ & \left(\frac{v_{h}^{n+1}-v_{h}^{n}}{\Delta t}+\theta \operatorname{div}_{h}^{n+1}+(1-\theta) \operatorname{div} \mathbf{q}_{h}^{n}-\theta f^{n+1}-(1-\theta) f^{n},\right. \\
& \left.\frac{v_{h}^{n+1}-v_{h}^{n}}{\Delta t}+\theta \operatorname{div} \mathbf{q}_{h}^{n+1}+(1-\theta) \operatorname{div} \mathbf{q}_{h}^{n}-\theta f^{n+1}-(1-\theta) f^{n}\right) \\
+ & \left.\left(\mathbf{k} \nabla v_{h}^{n+1}+\mathbf{q}_{h}^{n+1}, \mathbf{k} \nabla v_{h}^{n+1}+\mathbf{q}_{h}^{n+1}\right)\right] .
\end{aligned}
$$

Pode-se observar que para $\theta=0.5$ obtém-se um esquema do tipo Crank Nicolson e para $\theta=1.0$, um esquema totalmente implícito. A minimização do funcional (4.3) em relação a $v_{h}^{n+1}$ e a $\mathbf{q}_{h}^{n+1}$ conduz ao seguinte problema variacional:

Problema $V 3_{h}$ : Para $n=0,1, \ldots, N-1$, encontrar $\left(\mathbf{p}_{h}, u_{h}\right)_{n} \in V_{h} \times U_{h}$, onde $V_{h} \subset V$ e $U_{h} \subset U$, tal que:

$$
B_{n}\left\{\left(\mathbf{p}_{h}, u_{h}\right)_{n},\left(\mathbf{q}_{h}, v_{h}\right)_{n}\right\}=L_{n}\left(\mathbf{q}_{h}, v_{h}\right)_{n}
$$

onde

$$
\begin{aligned}
& B_{n}\left\{\left(\mathbf{p}_{h}, u_{h}\right)_{n},\left(\mathbf{q}_{h}, v_{h}\right)_{n}\right\}=\left(\frac{u_{h}^{n+1}}{\Delta t}\right.\left.+\theta \operatorname{div} \mathbf{p}_{h}^{n+1}, \frac{v_{h}^{n+1}}{\Delta t}+\theta \operatorname{div} \mathbf{q}_{h}^{n+1}\right) \\
&+\left(\mathbf{k} \nabla u_{h}^{n+1}+\mathbf{p}_{h}^{n+1}, \mathbf{k} \nabla v_{h}^{n+1}+\mathbf{q}_{h}^{n+1}\right), \\
& L_{n}\left(\mathbf{q}_{h}, v_{h}\right)_{n}=\left(\frac{u_{h}^{n}}{\Delta t}+(1-\theta) \operatorname{div} \mathbf{p}_{h}^{n}+\theta f^{n+1}+(1-\theta) f^{n}, \frac{v_{h}^{n+1}}{\Delta t}+\theta \operatorname{div} \mathbf{q}_{h}^{n+1}\right) .
\end{aligned}
$$

Com base nos resultados encontrados para o problema estacionário, acrescentou-se a condição de irrotacionalidade do fluxo no tempo $t_{n+\theta}$ a esta formulação. Dessa forma, define-se o novo funcional de mínimos quadrados utilizando-se a aproximação $\operatorname{rot} \mathbf{q}_{h}^{n+\theta}=\theta \operatorname{rot} \mathbf{q}_{h}^{n+1}+(1-\theta) \operatorname{rot} \mathbf{q}_{h}^{n}$, resultando em:

$$
\bar{J}\left(\mathbf{q}_{h}, v_{h}\right)=J\left(\mathbf{q}_{h}, v_{h}\right)+\frac{1}{2} \int_{\Omega}\left(\theta \operatorname{rot}_{h}^{n+1}+(1-\theta) \operatorname{rot} \mathbf{q}_{h}^{n}\right)^{2} d \Omega .
$$

Então, o problema variacional resultante da minimização do funcional (4.5) com relação a $v_{h}^{n+1}$ e a $\mathbf{q}_{h}^{n+1}$ é dado por: 
Problema $V 4_{h}$ : Para $n=0,1, \ldots, N-1$ encontrar $\left(\mathbf{p}_{h}, u_{h}\right)_{n} \in V_{h} \times W_{h}$, onde $V_{h} \subset V$ e $W_{h} \subset W$, tal que:

$$
\bar{B}_{n}\left\{\left(\mathbf{p}_{h}, u_{h}\right)_{n},\left(\mathbf{q}_{h}, v_{h}\right)_{n}\right\}=\bar{L}_{n}\left(\mathbf{q}_{h}, v_{h}\right)_{n},
$$

onde

$$
\begin{gathered}
\bar{B}_{n}\left\{\left(\mathbf{p}_{h}, u_{h}\right)_{n},\left(\mathbf{q}_{h}, v_{h}\right)_{n}\right\}=B_{n}\left\{\left(\mathbf{p}_{h}, u_{h}\right)_{n},\left(\mathbf{q}_{h}, v_{h}\right)_{n}\right\}+\left(\theta \operatorname{rot} \mathbf{p}^{n+1}, \theta \operatorname{rot} \mathbf{q}^{n+1}\right), \\
\bar{L}_{n}\left(\mathbf{q}_{h}, v_{h}\right)_{n}=L_{n}\left(\mathbf{q}_{h}, v_{h}\right)_{n}+\left((1-\theta) \operatorname{rot} \mathbf{p}^{n}, \theta \operatorname{rot}^{n+1}\right) .
\end{gathered}
$$

\subsection{Resultados Numéricos}

Como exemplo numérico utilizado para testar a convergência das formulações propostas, tomou-se um problema definido no domínio $[0,1] \times[0,1]$ com solução exata $u=e^{-k \pi^{2} t} \operatorname{sen} \pi x \operatorname{sen} \pi y$. Foram utilizados elementos isoparamétricos bilineares com interpolação de igual ordem $(l=k=1)$ e malhas de $8 \times 8,16 \times 16,32 \times 32$ e $64 \times 64$ elementos e $\Delta t=\Delta x$. Na Figura 2, apresentam-se resultados das taxas de convergência para a formulação totalmente implícita $(\theta=1) \mathrm{em} t=5$. Na Figura 3 , encontram-se resultados da formulação ponderada $(\theta=0.5)$ com e sem a inclusão da condição de irrotacionalidade do fluxo.

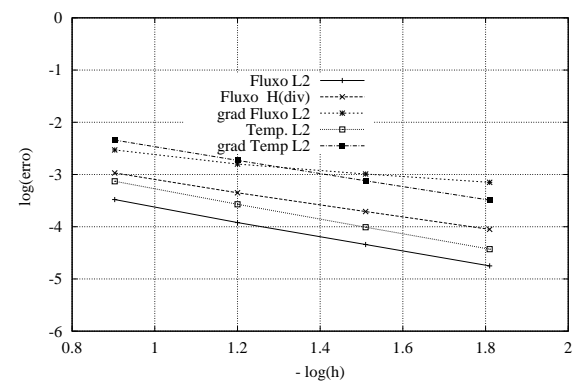

(a)

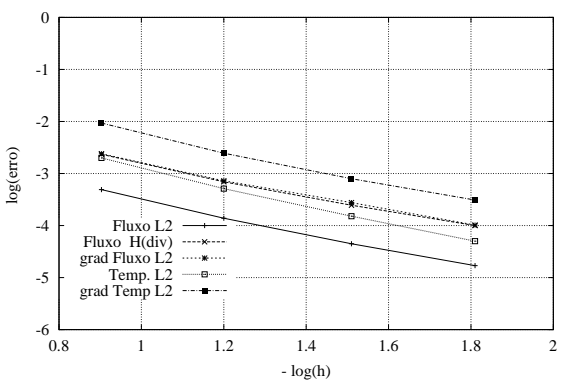

(b)

Figura 2: Formulação totalmente implícita (a) Formulação sem rotacional; (b) Formulação com rotacional

Pode-se observar que, mesmo para o problema transiente, o método converge quando aproximam-se $u$ e p com interpolações de igual ordem. A inclusão da condição de irrotacionalidade, também nestes casos, melhora as taxas de convergência para o fluxo, obtendo-se aproximações de igual ordem para as variáveis envolvidas no problema.

\section{Conclusões}

A formulação de mínimos quadrados é uma alternativa, quando se necessita trabalhar com formulações mistas. Neste caso, tanto para o problema estacionário 


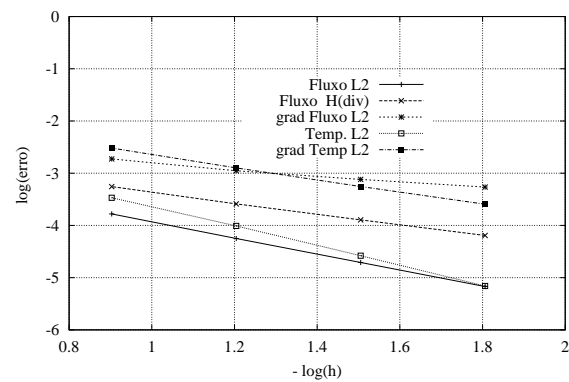

(a)

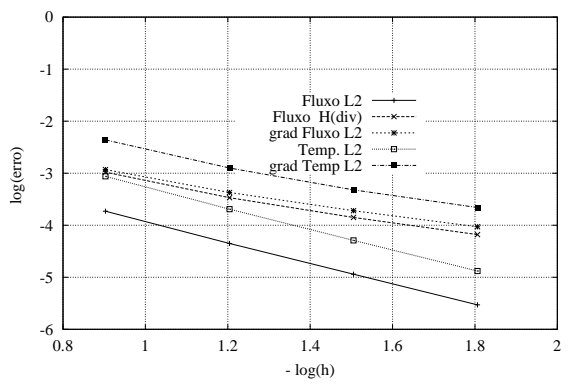

(b)

Figura 3: Formulação ponderada (a) Formulação sem rotacional; (b) Formulação com rotacional

quanto para o problema transiente, não há necessidade de compatibilidade entre os espaços de aproximação das variáveis escalar e vetorial, já que se pode acomodar interpolações de igual ordem. As formulações propostas mostram-se convergentes e constata-se, nos casos analisados, que tanto no problema estacionário quanto no problema transiente, a inclusão da parcela devida à irrotacionalidade do fluxo, que não altera o número de incógnitas do problema discretizado, melhora as taxas de convergência para essa variável.

A formulação ponderada, onde a equação evolutiva está aproximada no tempo $t_{n+\theta}$, com $\theta=0.5$, apresentou resultados mais precisos que a formulação totalmente implícita, embora esta apresente excelentes características de estabilidade.

Abstract. In this work we present least-squares finite element formulations for stationary and transient diffusion problems as alternatives to the classical Galerkin finite element formulation. For the transient case, we consider and propose semidiscrete mixed formulations in the same way as done with the stationary problem. Two kinds of time weighting are used and presented. The influence of the irrotationality condition on the convergence rates are shown and discussed for both cases.

\section{Referências}

[1] J. Bramble, A. Schatz, On the numerical solution of elliptic boundary-value problems by least-squares aproximation, em "Numerical Solution of P.D.E.", Vol. II, pp. 107-133, Academic Press, New York, 1970.

[2] Z. Cai, T.A. Manteuffel, S.F. Mccormick, First-order system least squares for second-order partial differential equations: Part II, SIAM J. Numer. Anal., 34 (1997), 425-454.

[3] G.F. Carey, "Finite Element Modeling of Environmental Problems", John Wiley and Sons, 1995. 
[4] G.F. Carey, B.N. Jiang, Least square finite element method and preconditioned conjugate gradient solution, Int. J. Numer. Met. Eng., 24 (1987), 1283-1296.

[5] T.F. Chen, Semidiscrete least-squares methods for linear convection-diffusion problem, Comput. Math. Applic., 24 (1992), 29-44 .

[6] V. Girault, P.A. Raviart, "Finite Element Methods for Navier-Stokes Equations", Springer-Verlag, 1986.

[7] B.N. Jiang, Optimal least-squares finite element method for elliptic problems. Comp. Meth. Appl. Mech. Eng., 102 (1998), 199-212.

[8] R.C.P. Leal-Toledo, M.F.S.S. Menna Barreto, E.M. Toledo, A finite element least squares formulation for stationary and transient advection-diffusion problems, em "Anais do XX Congresso Ibero - Latino - Americano de Métodos Computacionais em Engenharia", São Paulo, SP, 1999.

[9] R.C.P. Leal-Toledo, A.F.D. Loula, E.M. Toledo, N.F.F. Ebecken, Aplicação do método dos elementos finitos a uma formulação de mínimos quadrados, em "Anais do XIV Congresso Ibero - Latino - Americano de Métodos Computacionais em Engenharia", São Paulo, SP, 1993.

[10] R.C.P. Leal-Toledo, "Estudo de Uma Formulação de Mínimos Quadrados para o Método dos Elementos Finitos Mistos ", Tese de Doutorado, COPPE/UFRJ, Rio de Janeiro, RJ, 1992.

[11] A.I. Pehlivanov, G.F. Carey, R.D. Lazarov, Least squares mixed finite elements for second order elliptic problems, SIAM J. Numer. Anal., 31 (1994), 13681377.

[12] E.M. Toledo, " Novas Formulações Mistas de Elementos Finitos Mistos com Pós-processamento", Tese de Doutorado, COPPE/UFRJ, Rio de Janeiro, RJ, 1990. 